\title{
Modelado de la Cinética de Secado en Microondas de Ñame
}

\author{
Carlos García-Mogollon ${ }^{(1)^{*}}$, Mauricio Sierra-Bautista ${ }^{(2)}$ y Leonardo Miranda-Ramos ${ }^{(2)}$ \\ (1) Universidad de Sucre Facultad de Ingeniería, Sincelejo, Sucre-Colombia \\ (e-mail: carlos.garcia@unisucre.edu.co) \\ (2) Universidad de Antioquia. Facultad de Ingeniería, Medellín, Antioquia-Colombia \\ ${ }^{*}$ Autor a quien debe dirigirse la correspondencia
}

Recibido Ago. 14, 2015; Aceptado Oct. 5, 2015; Versión final Nov. 8, 2015, Publicado Feb. 2016

\begin{abstract}
Resumen
Se evaluó el efecto de la potencia de microondas y la masa en el secado de ñame (Dioscorea rotundata) de $1 \mathrm{~cm}$ de espesor y $3 \mathrm{~cm}$ de diámetro. Se sometieron $50 \mathrm{~g}, 60 \mathrm{~g}$ y $70 \mathrm{~g}$ de chips a potencias de $420 \mathrm{~W}$, 560W y 700W hasta la humedad de equilibrio. Se midieron el tiempo de secado, la cinética de secado, el coeficiente de difusividad térmica y la energía de activación. Los tratamientos mostraron un efecto altamente significativo de la potencia y la masa en el tiempo de secado $\mathrm{p}<0.05$ ). El modelo que mejor describe el comportamiento de la cinética de secado de ñame fue el modelo de Midilli and Kucuk (error cuadrático medio $<0,01$ y coeficiente de regresión lineal $>0.99$ ). Los tiempos de secado fueron reducidos cuando la intensidad del tratamiento fue mayor y el coeficiente de difusividad efectiva presentó valores entre $1.83 \times 10^{-4}$ y $9.41 \times 10^{-4} \mathrm{~m}^{2} / \mathrm{s}$ y una energía de activación de $22.38 \mathrm{~W} / \mathrm{g}$.
\end{abstract}

Palabras clave: Dioscorea rotundata; difusividad; deshidratación; energía de activación

\section{Modeling of Microwave Drying Kinetics of Yam}

\begin{abstract}
The effect of microwave power and mass in the drying of slices yam (Dioscorea rotundata) of $1 \mathrm{~cm}$ thick and $3 \mathrm{~cm}$ in diameter was evaluated. The chips were subjected to three different powers, $420 \mathrm{~W}, 560 \mathrm{~W}$ and $700 \mathrm{~W}$; with three different masses $50 \mathrm{~g}, 60 \mathrm{~g}$ and $70 \mathrm{~g}$. The drying time, the kinetics of drying, the coefficient of thermal diffusivity and the activation energy were measured. Treatments showed a highly significant effect of the power and the mass on drying time $(p<0.05)$. The Midilli and Kucuk's model is best to describe the drying kinetics (average squared error $<0.01$ and linear correlation coefficient $>0.99$ ) Drying times were reduced when treatment intensity was higher and the effective diffusivity coefficient presented values between $1.83 \times 10^{-4}$ to $9.41 \times 10^{-4} \mathrm{~m}^{2} / \mathrm{s}$ and activation energy of $22.38 \mathrm{~W} / \mathrm{g}$.
\end{abstract}

Keywords: Dioscorea rotundata; dehydration; diffusivity; activation energy 


\section{INTRODUCCIÓN}

El ñame (Dioscorea rotundata) es un alimento básico en muchas regiones tropicales, principalmente en el occidente de África, Asia, el Pacifico y el Caribe. En esta última región se haya la Costa Atlántica Colombiana principal productora nacional, debido que representa más del $90 \%$ de la producción nacional de ñame, mientras que otros departamentos como Antioquia, Chocó, Casanare y Vaupés tienen una participación minoritaria. En Colombia se cultivaron alrededor de 314.991 toneladas en 2013, con un rendimiento de 10.7 toneladas por hectárea sembrada (Agronet, 2015). Las preparaciones del ñame van desde lo salado hasta lo dulce, desde el tradicional mote de queso hasta el dulce de ñame, usualmente elaborado en época de semana santa. (Reina, 2012).

La deshidratación es un proceso que logra los objetivos de conservación a través de la reducción del contenido de agua y el decremento de los costos de transporte por la reducción de peso y volumen del alimento (Darvishi et al., 2014). El secado de ñame en las condiciones de humedad relativa (89\%) y alta humedad composicional (Garcia et al., 2012) hacen que el proceso de secado con aire caliente y al sol genere dificultades operativas en el deterioro del producto y largos tiempos de proceso (Ordóñez et al., 2014). El uso del microondas en secado resulta mucho más benéfico en la reducción del tiempo de proceso siendo más uniforme y más eficiente en el uso de la energía comparado con el secado con aire caliente natural o convectivo (Sarimeseli, 2011; Darvishi et al., 2013; Zarein et al., 2015).

El secado de productos alimenticios está controlado por fenómenos de trasferencia de masa y energía, que se describen usando modelos fenomenológicos como el de Newton, Page, Page modificado, Henderson y Pabis modificado, Verma et al., Two term exponential, Wang and Singh, Henderson y Pabis, Difusional, Midilli et al. y Logaritmico (Ajala et al., 2012; Salcedo et al., 2014; Torregroza et al., 2014) que permiten no solo predecir la cinética del proceso, el contenido final de humedad y los requisitos del proceso de secado de los productos hortofrutícolas; sino también los parámetros difusionales acorde a la ley de difusión de Fick en capa delgada para la simulación del secado por microondas (Rayaguru y Routray, 2011; Kumar et al., 2012; Ruiz et al., 2012; Minaei et al., 2012; Kahyaoglu et al., 2012). El modelado matemático del fenómeno de secado es necesario para su evaluación, determinación de parámetros y optimización, por ello, los modelos validados deben describir, cuantificar y predecir el proceso de secado (Ferreira y Costa, 2010; Erbay y Icier, 2010; Chen et al., 2013; Vasic et al., 2014). En este estudio se obtuvieron los parámetros cinéticos que modelaron el comportamiento de secado por microondas de los chips de ñame en las velocidades, tiempos y constantes cinéticas.

\section{MATERIALES Y MÉTODOS}

\section{Obtención y adecuación de la muestra}

Se tomaron muestras de ñame $(D$. rotundata) en el corregimiento de Berástegui, del municipio de Ciénaga de Oro del departamento de Córdoba, con coordenadas astronómicas de latitud norte $75^{\circ} 54^{\prime \prime}$ de longitud oeste de Greenwich de $8^{\circ} 30^{\prime \prime}$, con temperaturas que oscilan entre 35 y $38^{\circ} \mathrm{C}$ y presión atmosférica de 750 a 760 Torr. La adecuación se realizará para los chips de ñame dándole dimensiones de $3 \mathrm{~cm}$ de diámetro y $1 \mathrm{~cm}$ de espesor, caracterizando el contenido de humedad (AOAC 950,46).

\section{Procedimiento para el secado}

Para el secado en microondas se utilizó un horno microondas doméstico de marca Haceb HM-1.1 con una frecuencia de $2450 \mathrm{MHz}$ y dimensiones internas de $354 \times 228 \times 373 \mathrm{~mm}$. Se tomaron masas de 50,60 y $70 \mathrm{~g}$ de muestra y distribuyeron uniformemente en el plato del microondas y se evaluaron las potencias de $420 \mathrm{~W}$, $560 \mathrm{~W}$ y $700 \mathrm{~W}$. La masa de las muestras durante el secado se pesaron en una balanza analítica (MettlerPS4000 con exactitud $\pm 0.01 \mathrm{~g}$ ) cada 30 segundos para $420 \mathrm{~W}, 560 \mathrm{~W}$ y $700 \mathrm{~W}$. Las bandejas con las muestras fueron sacadas de la cámara de secado, pesadas y puestas de nuevo en la cámara.

\section{Modelamiento del secado por microondas}

Para la construcción de las curvas de secado se graficó el contenido de humedad del producto en base seca (MS) contra el tiempo para la determinación de las curvas de secado. Para determinar el modelo que mejor describe el comportamiento cinético de secado de ñame se utilizaron los modelos de Newton, Page, Page Modificado, Henderson y Pabis, Logaritmico, Thomson, Difusional, Dos términos, Midilli y Kucuk, Modificado de Henderson y Pabis, Two Term Exponential, Verma et al., Modified Page Equation-II en función de la razón de humedad (MR) (Ec. 1).

$M R=\left(X-X_{e}\right) /\left(X_{0}-X_{e}\right)$ 
Donde $X$ : el contenido de humedad en un tiempo específico, $X e$ : el contenido de humedad de equilibrio y Xo: el contenido inicial de humedad, todas expresadas en g agua/g MS. Se utilizó el método de regresión no lineal para estimar las constantes de los modelos.

Para el cálculo del coeficiente difusivo se utilizó el modelo difusional de la solución de la segunda ley de Fick para una geometría de lámina infinita y distribución uniforme de la humedad inicial (Ec. 2); de donde se toma solo el primer término de la ecuación.

$$
M R=\frac{8}{\pi^{2}} \sum_{n=1}^{\infty} \frac{1}{(2 n-1)^{2}} \exp \left[-(2 n+1)^{2} \frac{\pi^{2} D_{\text {eff }} t}{4 L^{2}}\right]
$$

Donde Deff : el coeficiente efectivo de difusividad de agua $\left(\mathrm{m}^{2} / \mathrm{s}\right)$, t: el tiempo (segundos), L: el espesor medio de la lámina finita $(\mathrm{m})$ y $\mathrm{n}$ : el número de series de la sumatoria. Para calcular la energía de activación se utilizó una modificación de la ecuación de Arrhenius (Ec. 3) donde existe una correlación entre la Deff y la densidad de carga $(\mathrm{m} / \mathrm{P})$ que relaciona la masa de la muestra con la potencia $(\mathrm{P})$ de salida de microondas en lugar de la temperatura del aire (Ozbek y Dadali 2007).

$D_{\text {eff }}=$ Do.exp $\left(-\frac{E_{a} m}{P}\right)$

Donde Do: factor pre-exponencial de Arrhenius ( $\left.\mathrm{m}^{2} / \mathrm{s}\right)$, Ea: energía de activación $(\mathrm{W} / \mathrm{g})$ y m: masa (g).

La suma de cuadrados del error (SSE) y el coeficiente de determinación $\left(R^{2}\right)$ fueron estimados para evaluar la falta de ajuste de los modelos. Bajos valores de SSE y altos de $\mathrm{R}^{2}$ se usaran como criterio para indicar el mejor ajuste del modelo.

\section{Diseño experimental}

Se aplicó un diseño experimental completamente al azar con arreglo factorial $3^{2}$ correspondiente a las variables numéricas potencia $(420,560$ y $700 \mathrm{~W})$ y masa de ñame $(50,60$ y $70 \mathrm{~g})$; con 3 repeticiones, para un total de 27 unidades experimentales; 3 repeticiones para un total de 27 unidades experimentales. Se aplicó el Test de Tukey con un nivel de significación de $p<0,05$ al tiempo de secado.

\section{RESULTADOS Y DISCUSIÓN}

\section{Cinética de secado}

En todos los tratamientos se evidencia una influencia altamente significativa $(p<0,05)$ de la potencia y la masa en el tiempo de secado. El contenido inicial de humedad en el ñame fresco fue de $2.09 \pm 0.08 \mathrm{~g}$ agua/gMS y el peso final fue de $16.5 \mathrm{~g} ; 19.1 \mathrm{~g}$ y $23.4 \mathrm{~g}$ para las muestras de 50,60 y $70 \mathrm{~g}$ respectivamente. Las curvas de secado obtenidas para las tres potencias (420W, 560W y $700 \mathrm{~W}$ ) muestran el efecto sobre el proceso (Figura 1, 2 y 3 ), ya que al aumentar la potencia disminuye el tiempo de secado para lograr una humedad de equilibrio de $0.002 \mathrm{~g}$ agua $/ \mathrm{g}$ MS, necesitando menos tiempo con $50 \mathrm{~g}$ a $420 \mathrm{~W}$ (26.0min) que para $560 \mathrm{~W}$ y $700 \mathrm{~W}$ con tiempos de $13.2 \mathrm{~min}$ y $10.0 \mathrm{~min}$ respectivamente. Los tiempos de secado para $60 \mathrm{~g}$ y $70 \mathrm{~g}$ se observa la misma tendencia en la reducción del tiempo, lo que representa que en general la transferencia de masa (agua) es más rápida cuando el peso de la muestra es menor y la intensidad de la potencia aumenta. En las curvas de secado se observa la tendencia exponencial de la cinética, validando a priori la utilización de modelos propuestos para simular todo el proceso de secado.

Estudios similares fueron hechos por Sagñay (2009), quien deshidrató frutilla (Fragaria vesca) por 14.5h a $100 \mathrm{~W}, 2.25 \mathrm{~h}$ a $200 \mathrm{~W}$ y $1.16 \mathrm{~h}$ a $300 \mathrm{~W}$; espinacas en microondas de $2450 \mathrm{MHz}$ con tiempos de $65.0,15.0$ y 8.0 min a una potencia del $120 \mathrm{~W}, 420 \mathrm{~W}$ y $700 \mathrm{~W}$ respectivamente (Mackeprang et al., 2010); secado al vacío de hojas de menta por 13.0 min a 1600W, 10 min a 2240W (Therdthai y Zhou, 2009), secado de chips de yuca en microondas con tiempos de 124, 70 y 30 min a potencia de 140, 280 y 560W (Castelar, 2013). Los resultados obtenidos indican que la transferencia de masa asociada a la perdida de agua en los chips de ñame fue más rápida con la mayor potencia en el microonda, dado que genera mayor calor en la muestra, creando una mayor diferencia de presión de vapor entre el centro y la superficie del chips debido al calentamiento. Estos resultados son similares a los reportados por mecanismo de deshidratación diferentes como la liofilización (Serna et al., 2015), aire caliente (Montes et al., 2008; Michalewicz et al., 2011; Salcedo et al., 2014), radiación solar directa (Machado et al., 2010; Banout et al., 2011). 


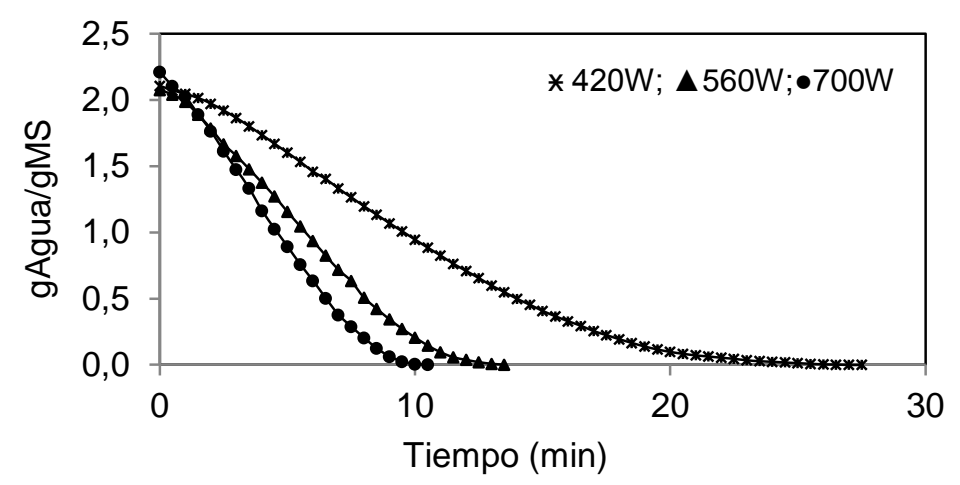

Fig. 1: Rapidez de secado para una masa de $50 \mathrm{~g}$

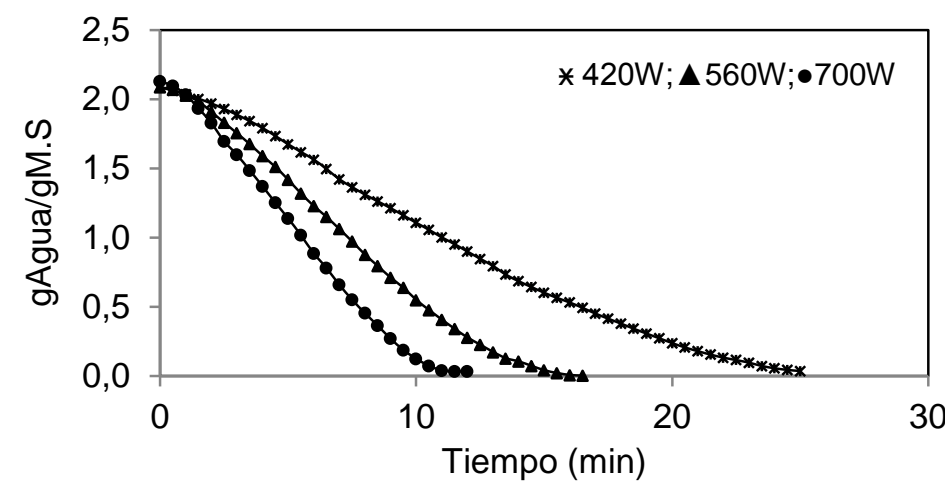

Fig. 2: Rapidez de secado para una masa de $60 \mathrm{~g}$

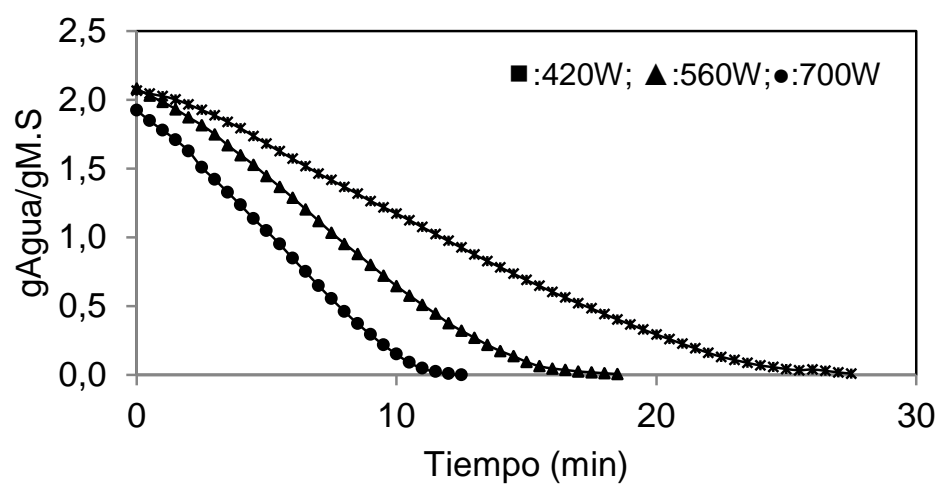

Fig. 3: Rapidez de secado para una masa de $70 \mathrm{~g}$

\section{Modelado Matemática}

Los modelos de Midilli and Kucuk, Page y Modified Page Equation II, describen satisfactoriamente la cinética de secado de los chips de ñame con valores de $R^{2}>99 \%$ y SSE $<1 \%$ en la mayoría de los casos. Sin embargo, la mejor explicación al comportamiento de secado es el modelo de Midilli and Kucuk (Ec. 4). A través de la regresión no lineal utilizada para determinar los parámetros $k(1 / \mathrm{min}), n$, a y $b$ del modelo de Midilli and Kucuk con $\mathrm{R}^{2}>99.40$ y $0.01<\mathrm{SSE}<0.00$ se pudo demostrar que es adecuado para describir la cinética de secado de los chips de ñame.

$$
M R=a\left[\operatorname{Exp}\left(-k t^{n}\right)\right]+b t
$$

El parámetro $k$ aumenta con el incremento de la potencia, es por ello que para una potencia de $420 \mathrm{~W}$ y $50 \mathrm{~g}$ el parámetro tuvo un valor de $19.59 \mathrm{~min}^{-1}$, mucho menor que los valores de $k$ para la potencia $560 \mathrm{~W}$ y $700 \mathrm{~W}$, los cuales fueron de $71.94 \mathrm{~min}^{-1}$ y $104.96 \mathrm{~min}^{-1}$ respectivamente, describiendo una mayor pendiente que indica una mayor velocidad de secado (Tabla 1). De igual forma a medida que aumenta la masa los valores de $k$ disminuyen. Por su parte el parámetro $n$ es mayor a uno, indicando que la relación entre MR y el 
tiempo no sigue una cinética de primer orden. Resultados en otros estudios con hortofrutícolas que mejor describieron el secado en microondas (180 a 540W) el modelo de Midilli et al. (Darvishi et al., 2014); con aire caliente el modelo Logarítmico en ñame (Montes et al., 2008), en cajuil el modelo Modified Page equation II, Werma et al. y Logarítmico (Michalewicz et al., 2011).

Tabla 1. Valores medios de los parámetros del modelo de Midilli and Kucuk

\begin{tabular}{|c|c|c|c|c|c|c|c|c|c|c|c|c|}
\hline Potencia & \multicolumn{5}{|c|}{$50 \mathrm{~g}$} & \multicolumn{5}{|c|}{$60 \mathrm{~g}$} & \multicolumn{4}{|c|}{$70 \mathrm{~g}$} \\
\hline $\mathrm{W}$ & $\mathrm{k}$ & $\mathrm{n}$ & $\mathrm{a}$ & $\mathrm{b}$ & $\mathrm{K}$ & $\mathrm{n}$ & $\mathrm{a}$ & $\mathrm{b}$ & $\mathrm{k}$ & $\mathrm{n}$ & $\mathrm{a}$ & $\mathrm{b}$ \\
\hline 420 & 19,59 & 1,78 & 0,977 & 0 & 14,33 & 1,75 & 0,97 & 0 & 14,52 & 1,840 & 0,968 & 0 \\
\hline 560 & 71,94 & 1,95 & 0,968 & 0 & 48,4 & 1,99 & 0,97 & 0 & 41,00 & 1,978 & 0,956 & 0 \\
\hline 700 & 104,9 & 1,90 & 0,96 & 0 & 87,52 & 1,99 & 0,97 & 0 & 78,43 & 1,979 & 0,947 & 0 \\
\hline
\end{tabular}

\section{Coeficiente de difusividad efectiva}

Los valores de difusividad fueron mayores a medida que la potencia y la masa aumentaron (Tabla 2), lo que demuestra una disminución de las resistencias internas del sólido. Los valores de difusividad en alimentos oscila en forma general de $10^{-6}$ a $10^{-11} \mathrm{~m}^{2} / \mathrm{s}$ (Zarein et al., 2015), mientras que los obtenidos están en valores de $10^{-3}$ a $10^{-4} \mathrm{~m}^{2} / \mathrm{s}$. Una explicación de ese aumento se debe al menor encogimiento observado en los chips cuando fueron sometidos a potencias más altas; menor encogimiento implica una mayor porosidad y por ende una mayor área de transferencia de masa. Estudios previos en secado convectivo de manzanas,

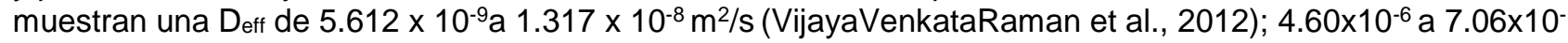
${ }^{6} \mathrm{~m}^{2} / \mathrm{s}$ en liofilización de cubos de manzana (Kahyaoglu et al., 2012), en rodajas de ñame secadas a $70^{\circ} \mathrm{C}$ un Deff de $4.91 \times 10^{-6} \mathrm{~m}^{2} / \mathrm{s}$ (Montes et al., 2008), en yuca de $1,261 \times 10^{-10}$ a $4,137 \times 10^{-10} \mathrm{~m}^{2} / \mathrm{s}$ (Salcedo et al., 2014). En productos derivados de tubérculos se ha reportado valores inferiores de $D_{\text {eff }}$ a temperaturas más altas en el secado convectivo (Fernando et al., 2011; Ajala et al., 2012) explicando que la variabilidad de la difusividad efectiva depende del mecanismo de calentamiento, en el caso del calentamiento por microondas favorece la operación de secado comparado con el convectivo.

Tabla 2. Difusividad efectiva

\begin{tabular}{|c|c|c|c|}
\hline Potencia $(\mathrm{W})$ & Densidad de carga $(\mathrm{g} / \mathrm{W})$ & $D_{\text {eff }}\left(\mathrm{m}^{2} / \mathrm{s}\right)$ & Desviación estándar \\
\hline 420 & $0,14 \pm 0,023$ & $1,83 \times 10-4$ & 0,0000409 \\
\hline 560 & $0,10 \pm 0,017$ & $5,71 \times 10-4$ & 0,0000548 \\
\hline 700 & $0,08 \pm 0,014$ & $9,41 \times 10-4$ & 0,0001871 \\
\hline
\end{tabular}

La relación de la difusividad efectiva con la potencia (Ec. 5) nos muestra una ecuación de primer orden con un $\mathrm{R}^{2}$ de 0,99 .

$$
D_{\text {eff }}=3 \times 10^{-6} \mathrm{P}-1 \times 10^{-3}
$$

Se puede observar como la $D_{\text {eff }}$ aumenta con el incremento de la densidad de carga, esto se explica por el aumento de la energía aprovechada en calentamiento, lo cual incrementa la actividad de las moléculas de agua conduciendo a la mayor difusividad del agua cuanto los chips de ñame son sometidos a una mayor potencia de microondas. La dependencia de la difusividad efectiva con la masa y la potencia (Ozbek y Dadali 2007) fue evaluada usando los modelos exponencial $\left(R^{2}=0,81\right)$ y lineal $\left(R^{2}=0,93\right)$. El modelo exponencial (Ec. 6) nos permite conocer la energía de activación (Ea) y el valor pre-exponencial del modelo de Arrhenius $\left(D_{0}\right)$.

$$
D_{\text {eff }}=0,00004 e^{22,38 \frac{m}{p}}
$$

Donde $D_{0}$ es 0.00004 y Ea es $22.38 \mathrm{~W} / \mathrm{g}$, el cual es comparativamente superior a los reportados por Darvishi et al. (2013) de $11.14 \mathrm{~W} / \mathrm{g}$ en sardinas, Zarein et al. (2015) de $12.15 \mathrm{~W} / \mathrm{g}$ en manzanas, de 6.92 $\mathrm{W} / \mathrm{g}$ en yuca (Castelar, 2014), de $16.67 \mathrm{~W} / \mathrm{g}$ y $24.22 \mathrm{~W} / \mathrm{g}$ para granada dulce y amarga respectivamente (Minaei et al., 2012) y de $13.6 \mathrm{~W} / \mathrm{g}$ para hoja de pandanus (Rayaguru y Routray, 2011), 


\section{CONCLUSIONES}

En el secado de chips de ñame por microondas a medida que la intensidad del tratamiento aumenta la velocidad de secado es mayor y los tiempos de secado disminuyen, siendo el tiempo de secado más corto para el tratamiento con masa de $50 \mathrm{~g}$ y potencia de $700 \mathrm{~W}$ y el tiempo de secado más largo para una masa de $70 \mathrm{~g}$ y potencia de $420 \mathrm{~W}$.

De los modelos estudiados el que mejor se ajustó al comportamiento de secado en todos los tratamientos realizados fue el modelo de Midilli and Kucuk, sin embargo otros modelos como page, page modificado ecuación II, logarítmico, dos términos, entre otros pueden describir fácilmente la cinética de secado de los chips de ñame en microondas. Los valores de $\mathrm{k}$ y $\mathrm{n}$ del modelo permiten establecer que la pérdida de agua no sigue una cinética de primer orden.

El valor de la Difusividad efectiva se incrementa con el incremento de la energía aprovechada en calentamiento, lo cual incrementa la actividad de las moléculas de agua conduciendo a la mayor difusividad del agua cuanto los chips de ñame son sometidos al mecanismo de calentamiento por microondas.

\section{REFERENCIAS}

Agronet, Estadísticas Agroforestales Consolidado Nacional (2013), http://www.agronet.gov.co/agronetweb1/Estad\%C3\%ADsticas.aspx. Acceso: 20 de julio (2015)

Ajala, A. y otros tres autores, Drying Characteristics and Mathematical Modelling of Cassava Chips, Chemical and Process Engineering Research, 4, 1-9 (2012)

A.O.A.C.: Association of Official Analytical Chemist, Official Methods of Analysis, Section 954.10. 13th. Ed. Washington D.C., USA (1993)

Banout, J. y otros cinco autores, Design and performance evaluation of a Double-pass solar drier for drying of red chilli (Capsicum annum L.), doi:10.1016/j.solener.2010.12.017, Solar Energy, 85, 506-515 (2011)

Castellar, R., Evaluación de la cinética de rehidratación y cambio de color en chips de yuca (manihot esculenta, crantz) secadas en horno microondas, Trabajo de grado, Universidad de Córdoba, Ingeniero de Alimentos, Montería (2013)

Chen, D., Y. Zheng y X. Zhu, In-depth investigation on the pyrolysis kinetics of raw biomass. Part I: Kinetic analysis for the drying and devolatilization stages, Bioresource Technology, 131, 40-46 (2013)

Darvishi, H. y otros tres autores. Drying characteristics of sardine fish dried with microwave heating, Journal of the Saudi Society of Agricultural Sciences, 12, 121-127 (2013)

Darvishi, H. y otros cinco autores, Study of the drying kinetics of pepper, Journal of the Saudi Society of Agricultural Sciences, 13, 130-138 (2014)

Erbay, Z. y F. Icier, A review of thin layer drying of foods: theory, modeling, and experimental results, Critical Reviews in Food Science and Nutrition, 50, 441-464 (2010)

Fernando, W. J. N., H. C. Low y A. L. Ahmad, Dependence of the effective diffusion coefficient of moisture with thickness and temperature in convective drying of sliced materials. A study on slices of banana, cassava and pumpkin, Journal of Food Engineering, 102, 310-316 (2011)

Ferreira, S. y A. Costa, Parámetros de Transferencia de Materia en el Secado de Frutas, sin Necesidad de Datos de Disminución de Volumen, doi:10.1612/inf.tecnol.4360it.09, Información Tecnológica, 21(5), 87-98 (2010)

García, C., S. Dussan y N. Gutiérrez, Uso de horno microondas en la determinación de contenido de humedad: Yuca, Name y Plátano, Revista biotecnología en el sector agropecuario y agroindustrial, 10(1), 60-65 (2012)

Kahyaoglu, L., S. Sahin y G. Sumnu, Spouted bed and microwave-assisted spouted bed drying of parboiled wheat, Food and Bioproducts Processing, 90(2), 301-308 (2012) 
Kumar, C. y otros cinco autores, Multiphysics modelling of convective drying of food materials, Proceedings of the Global Engineering, Science and Technology Conference, Global Institute of Science and Technology, 1-13, Dhaka-Bangladesh, 28 a 29 de Diciembre (2012)

Machado, A. y otros tres autores, Estudio del Secado de Anacardo (Anacardium occidentale L.) mediante secador solar de radiación directa, Información Tecnológica, 21(1), 31-37 (2010)

Mackeprang, M., C. Paola y L. Campañone, Estudio experimental de la deshidratación de espinacas empleando microondas y energía solar, II Reunión Interdisciplinaria de Tecnología y Procesos Químicos, Huerta Grande - Córdoba - Argentina 2010

Michalewicz, J., J. Henriquez y J. Charamba, Secado de Cajuil (anacardium occidentale I.): Estudio Experimental y Modelado de la Cinética de Secado, Información Tecnológica, 22(6), 63-74 (2011)

Minaei, S. y otros tres autores, Mathematical models of drying pomegranate arils in vacuum and microwave dryers, J. Agri. Sci. Technol., 14, 311-325 (2012)

Montes, E. y otros cinco autores, Modelado de la cinética de secado de ñame (dioscorea rotundata) en capa delgada, Revista Ingeniería e Investigación, 28(2), 45-52 (2008)

Ordóñez, M., M. Gely y A. Pagano, Estudio de las propiedades físicas y de la cinética de secado de granos de maíz colorado duro, Avances en Ciencias e Ingeniería, 3(3), 153-171 (2012)

Ozbek, B. y G. Dadali, Thin-layer drying characteristics and modelling of mint leaves undergoing microwave treatment. J. Food Eng., 83, 541-549 (2007)

Rayaguru, K. y W. Routray, Microwave drying kinetics and quality characteristics of aromatic Pandanus amaryllifolius leaves, Int. Food Res. J., 18(3), 1035-1042 (2011)

Reina, Y., El cultivo del ñame en Colombia, Documentos de trabajo sobre economía regional, Banco de la República, Centro de estudios regionales, Cartagena, 168, 2-21 (2012)

Ruiz, I. y otros cuatro autores, Analytical model for variable moisture diffusivity estimation and drying simulation of shrinkable food products, Journal of Food Engineering, 108, 427-435 (2012)

Sagñay, N., Control de calidad de frutilla (fragaria vesca) deshidratada por método de microondas a tres potencias, Trabajo de grado, Escuela Superior Politécnica de Chimborazo, Riobamba-Ecuador, 38-42 (2009)

Salcedo, J. y otros cuatro autores, Cinética de secado de la yuca (Manihot esculenta Crantz) variedad CORPOICA M-tai en función de la temperatura y de la velocidad de aire, Rev. Ion., 27, 29-42 (2014)

Sarimeseli, A., Microwave drying characteristics of coriander (Coriandrum sativum L.) leaves, Energy Convers. Manag., 52, 1449-1453 (2011)

Serna, L., C. Torres y A. Ayala, Efecto de la Adición de Edulcorantes no Calóricos sobre las Propiedades Fisicoquímicas y la Cinética de Secado de Cáscara de Mango Liofilizado, doi: 10.4067/S071807642015000400006, Información Tecnologica, 26(4), 37-44 (2015)

Therdthai, N. y W. Zhou, Characterization of microwave vacuum drying and hot air drying of mint leaves (MenthacordifoliaOpiz ex Fresen), Journal of FoodEngineering, 91, 482-489 (2009)

Torregroza, A., y otros tres autores, Modelado de las cinéticas de secado de tres variedades de yuca industrial, DYNA, 81(186), 184-192 (2014)

Zarein, M., S. Hashem y B. Ghobadian, Investigation of microwave dryer effect on energy efficiency during drying of apple slices, Journal of the Saudi Society of Agricultural Sciences, 14(1), 41-47 (2015)

Vasic, M., Z. Grbavcic y Z. Radojevic, Determination of the moisture diffusivity coefficient and mathematical modeling of drying. Chemical Engineering and Processing, 76, 33- 44 (2014)

VijayaVenkataRaman, S., S. Iniyan y R. Goic, A review of solar drying technologies, Renewable and Sustainable Energy Reviews, 16(5), 2652-2670 (2012) 
